\title{
Mapping local microstructure and mechanical performance around carbon nanotube grafted silica fibres: methodologies for hierarchical composites
}

\author{
Hui Qian ${ }^{1,2,3}$, Gerhard Kalinka ${ }^{4}$, K.L. Andrew Chan ${ }^{5}$, Sergei G. Kazarian ${ }^{5}$, \\ Emile S. Greenhalgh ${ }^{1}$, Alexander Bismarck ${ }^{1,3, *}$, Milo S.P. Shaffer ${ }^{1,2, *}$
}

\footnotetext{
${ }^{1}$ The Composites Centre, Imperial College London, London SW7 2AZ, UK

${ }^{2}$ Department of Chemistry, Imperial College London, London SW7 2AZ, UK

${ }^{3}$ Polymer and Composite Engineering (PaCE) Group, Department of Chemical Engineering, Imperial College London, London SW7 2AZ, UK

${ }^{4}$ BAM Federal Institute for Materials Research and Testing, Berlin, D-12205, Germany

${ }^{5}$ Department of Chemical Engineering, Imperial College London, London SW7 2AZ, UK
}

\section{Table of Contents Graphic}

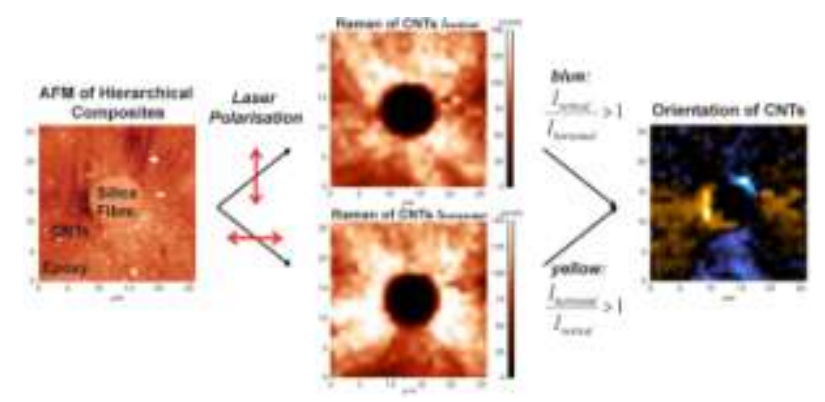

This paper presents a methodology for studying the localised microstructure and mechanical properties of hierarchical composites containing CNT-grafted fibres.

\footnotetext{
Corresponding author. Tel.:+44(0)20 7594 5578; Fax.:+44(0)20 $7594 \quad 5638$; E-mail address: a.bismarck@Imperial.ac.uk (A. Bismarck); Tel.:+44(0)20 7594 5825; Fax.:+44(0)20 7594 5801; E-mail address: m.shaffer@Imperial.ac.uk (M. S. P. Shaffer)
} 


\begin{abstract}
The introduction of carbon nanotubes (CNTs) modifies bulk polymer properties, depending on intrinsic quality, dispersion, alignment, interfacial chemistry and mechanical properties of the nanofiller. These effects can be exploited to enhance the matrices of conventional microscale fibre-reinforced polymer composites, by using primary reinforcing fibres grafted with CNTs. This paper presents a methodology that combines atomic force microscopy, polarised Raman spectroscopy, and nanoindentation techniques, to study the distribution, alignment and orientation of CNTs in the vicinity of epoxy-embedded micrometre-scale silica fibres, as well as, the resulting local mechanical properties of the matrix. Raman maps of key features in the CNT spectra clearly show the CNT distribution and orientation, including a 'parted' morphology associated with long grafted CNTs. The hardness and indentation modulus of the epoxy matrix were improved locally by $28 \%$ and $24 \%$, respectively, due to the reinforcing effects of CNTs. Moreover, a slower stress relaxation is observed in the epoxy region containing CNTs, which may be due to restricted molecular mobility of the matrix. The proposed methodology is likely to be relevant to further studies of nanocomposites and hierarchical composites.
\end{abstract}




\section{Introduction}

Owing to their extraordinary intrinsic mechanical properties, high aspect ratios and low density, carbon nanotubes (CNTs) are considered as an ideal candidate as nanoscale reinforcement for polymer matrix composite materials. ${ }^{1,2}$ In addition, exploitation of the excellent thermal and electrical conductivity of CNTs could lead to a new generation of multifunctional structural composite materials, which have potential applications in a wide range of fields. ${ }^{3}$ The processing, characterisation, and modelling of CNT-reinforced polymer nanocomposites has been studied extensively, with promising results reported for composite mechanical, thermal, and electrical properties. ${ }^{1,4}$ However, critical challenges, such as the dispersion and controlled alignment of the CNTs in the polymer matrix, still have to be addressed to maximise the potential advantages. ${ }^{5}$

More recently, interest has rapidly expanded in the use of CNTs within conventional fibrereinforced polymer composites, which are widely used in aerospace and the oil/gas industries, due to their light weight and excellent in-plane mechanical performance. ${ }^{6}$ The nanoscale CNTs are utilised alongside the microscale fibres, forming a hierarchical reinforcement microstructure. $^{7,8}$ The aim is to exploit the reinforcing capability of CNTs, which has demonstrated in nanocomposites, to improve the matrix-dominated properties of conventional composites; specifically, the focus is to address common problems such as delamination resistance, longitudinal compression and through-thickness performance. One of the possible routes to realise hierarchical composites is to grow CNTs directly onto fibre surfaces, creating 'hairy fibres'., 9-11 The advantages of this approach include increased primary fibre surface area, as well as mechanical interlocking and/or local stiffening in the fibre/matrix interphase, all of which may improve stress transfer and interfacial properties. Significant improvements in the interfacial shear strength of the model hierarchical composites containing CNT-grafted carbon $^{10,12-14}$ and silica ${ }^{15}$ fibres have already been reported. In addition, the grafting approach has the potential to provide high loadings of radially-orientated, well-spaced CNTs around the 
fibres, which could address traditional nanocomposite limitations associated with dispersion and alignment. The radial arrangement of nanofiller is appealing as a potentially ideal approach to increasing transverse properties, maximising interfacial toughness, and providing lateral support for the load-bearing microscale fibres in compression. However, no experimental data have yet demonstrated the local reinforcing effect of CNTs in the vicinity of fibres. In addition, new tools are needed for the study of the distribution and alignment of CNTs within hierarchical composites and nanocomposites, particularly after processing.

Polarised Raman spectroscopy is a non-destructive means to quantify the CNT orientation in nanocomposites, since the Raman intensity of CNTs is strongly orientation dependent; specifically, the Raman signal intensity decreases with increasing angle between the CNT and the polarisation direction of the incident laser beam. ${ }^{16,17}$ Instrumented nanoindentation provides an efficient means to assess the mechanical characteristics of CNT nanocomposites at a high spatial resolution, ${ }^{18}$ as has been demonstrated for both thermoset ${ }^{19-22}$ and thermoplastic $^{18,23}$ systems. This paper presents a new approach, combining atomic force microscopy (AFM), polarised Raman spectroscopy and nanoindentation techniques, to investigate the distribution, orientation, and mechanical influence of CNTs grafted onto microscale-fibres, embedded within an epoxy matrix. The cross-sectional surface of the composite specimens, containing CNT-grafted silica fibres, was initially examined using AFM and polarised Raman spectroscopy for obtaining maps of CNT distribution and orientation. Nanoindentation was then applied to the same area of the specimen to determine the hardness, indentation modulus and relaxation behaviour of the CNT-modified region of the epoxy matrix in the vicinity of the primary fibres. These factors are important in determining the overall performance of the macroscopic hierarchical composites.

\section{Experimental Section}

\subsection{Growth of CNTs on fibres}


The growth of CNTs on silica fibres (average diameter: $9 \mu \mathrm{m}$, Silfa, Albert Hellhake GmbH, Dortmund, Germany) was carried out using the injection chemical vapour deposition method. A detailed description of the growth process has been reported previously. ${ }^{11}$ Briefly, a feed precursor solution consisting mixture of $3 \mathrm{wt} . \%$ ferrocene, $58.5 \mathrm{wt} . \%$ toluene and $38.5 \mathrm{wt} . \%$ pyrazine was used for the growth of nitrogen-doped CNTs. The addition of pyrazine precursors has been shown to improve the straightness and packing of CNTs. ${ }^{24}$ The growth reaction was performed at $760{ }^{\circ} \mathrm{C}$ for $1 \mathrm{~h}$. SEM characterisation was performed on silica fibres (uncoated) before and after the growth using a field emission gun SEM (Gemini LEO 1525 FEG-SEM, Carl Zeiss NTS GmbH, Oberkochen, Germany), operating at $5 \mathrm{kV}$.

\subsection{Preparation of cross-sections of model hierarchical composites containing CNT- grafted silica fibres}

To fabricate model composite specimens, CNT-grafted fibres were laid flat into a $20 \mathrm{~mm}$ long, $5 \mathrm{~mm}$ wide and $10 \mathrm{~mm}$ high aluminum mould; epoxy (bisphenol-a resin with amine hardener, Epoxicure, Buehler GmbH, Düsseldorf, Germany) was introduced and cured at room temperature for $24 \mathrm{~h}$. Cross-sections perpendicular to the fibres were ground and polished with SiC grinding paper (P320, P800, P1200 and P4000, Struers Ltd., Rotherham, $\mathrm{UK}$ ) and $1 \mu \mathrm{m}$ polycrystalline diamond suspension (dye-free, Buehler GmbH, Düsseldorf, Germany), respectively, using a Buehler METASERV®2000 grinder/polisher.

\subsection{AFM characterisation of cross-sections of model hierarchical composites}

AFM experiments were performed using a standard silicon cantilever with a resonant frequency at $300 \mathrm{kHz}$ (Tetra tips, Olympus Ltd., Essex, UK) using a NTEGRA spectra system (NT-MDT Co., Moscow, Russia), which offers a unique integration of atomic force microscopy, confocal microscopy and Raman scattering spectroscopy. ${ }^{25}$ Topographic and phase images were recorded simultaneously in the tapping mode at a scanning speed of 1 line/s with 256 data points per line. Surface roughness analysis was conducted using the Nova analysis system by NT-MDT; three measurements were performed on each region and each 
measurement was based on a $2 \times 2 \mu \mathrm{m}^{2}$ area, containing 150 data points. The CNT/epoxy values were obtained based on the measurements conducted within the CNT-modified region identified next to the silica fibre.

\subsection{Raman characterisation of cross-sections of model hierarchical composites}

The polarised Raman study was conducted, at room temperature, using the NTEGRA Spectra system (NT-MDT Co., Moscow, Russia) equipped with a Renishaw Raman spectrometer (InVia, Renishaw Plc., Gloucestershire, UK) with a resolution of about $0.6 \mathrm{~cm}^{-1}$. More details about this system are provided in earlier work. ${ }^{25}$ A linearly polarised He-Ne laser (632.8 nm) was used as excitation source. The light was focused on the specimen surface with a long working distance x100 microscope objective (numerical aperture of 0.7 ). For the orientationdependent measurements, a half-wave retardation plate was inserted in front of the objective to rotate the polarisation of the incident laser beam. Raman scanning was conducted over a sample surface area of $26 \times 26 \mu \mathrm{m}^{2}$ at a $1 \mu \mathrm{m}$ step size and $10 \mathrm{~s}$ integration per spectrum; a total of 676 spectra were collected in the backscattered geometry. Mapping images of CNTs and polymer were then generated based on the maximum Raman intensity in the ranges of $1335-1355 \mathrm{~cm}^{-1}$ and $810-830 \mathrm{~cm}^{-1}$, respectively (refer to dark grey and light grey ranges in Figure 3), after subtracting the background signal.

\subsection{Nanoindentation on cross-sections of model hierarchical composites}

Nanoindentation tests were conducted at room temperature using a TS TriboScope (Hysitron Inc., Eden Prairie, USA) fitted with a diamond Berkovich indenter probe with a tip radius of approximately $20 \mathrm{~nm}$. For hardness and modulus determination, the samples were subjected to nanoindentation using a constant peak load of $1 \mathrm{mN}$. An array of $3 \times 20$ nanoindentations was selected, starting from the centre of silica fibre and terminating in the pure epoxy region (refer to Fig. 7a). A distance of $5 \mu \mathrm{m}$ was used between repeated nanoindentations to avoid the hardening effect or other influences from previous indents. ${ }^{26}$ Force versus displacement curves were recorded. The load and displacement resolutions of the system were $<1 \mathrm{nN}$ and 
$0.4 \mathrm{~nm}$, respectively. Based on the method proposed by Oliver and Pharr, ${ }^{27}$ the hardness of the test specimen, $H$, was determined through the equation:

$$
H=\frac{P_{\max }}{A}
$$

where $P_{\max }$ is the nanoindentation load and $A$ is the projected area of the hardness impression determined from the calibrated area function ${ }^{27}, A_{c}\left(h_{c}\right)=24.5 h_{c}^{2}+\sum_{i=0}^{n} c_{i+1} h_{c}^{\frac{1}{2^{i}}}$.

The relationship between the contact stiffness, $S$, and the reduced elastic modulus, $E_{r}$, is defined as follows: ${ }^{27}$

$$
S=\frac{d P}{d h}=\frac{2 \beta}{\sqrt{\pi}} \sqrt{A} E_{r}
$$

where $S$ is the contact stiffness obtained from the slope of the unloading curve, $\beta$ is a constant related to the indenter geometry $\left(\beta=1.034\right.$ for a Berkovich indenter $\left.{ }^{28}\right), E_{r}$ is the reduced elastic modulus due to a non-rigid indenter.

Since $E_{r}$ is a combination of the modulus of the specimen and the material of the indenter, the elastic modulus of the specimen can be calculated through the equation:

$$
\frac{1}{E_{r}}=\frac{1-v^{2}}{E}+\frac{1-v_{i n}^{2}}{E_{i n}}
$$

$E$ and $v$ are the elastic modulus and Poisson's ratio for the specimen, respectively, and $E_{\text {in }}$ and $v_{i n}$ are the same parameters for the material of the indenter. For a diamond indenter tip, $E_{\text {in }}$ is $1141 \mathrm{GPa}$ and $v_{\text {in }}$ is $0.07 .{ }^{29}$ The Poisson's ratios of the epoxy and silica were assumed to be 0.35 and 0.17 , respectively. ${ }^{30}$ Due to the radial arrangement of the CNT-grafting around the cylindrical fibre, the local CNT content, and hence the exact Poisson's ratio, may vary at different locations. However, the literature ${ }^{31,32}$ reports that CNT addition has only a minor effect on polymer Poisson's ratio (only a 3\% decrease in Poisson's ratio on the addition of 5\% CNTs). Even a 10\% reduction in Poisson's ratio would increase the calculated indentation 
modulus by less than $3 \%$, which is not significant in the current context. Thus, the same Poisson's ratio was used in the calculations for both the pure epoxy and CNT-modified epoxy regions. The mean and standard deviation, of hardness and modulus, at each distance, were calculated based on three independent indentation measurements.

\subsection{Stress relaxation testing on cross-sections of model hierarchical composites}

The stress relaxation tests were carried out using the same equipment as for the nanoindentation tests. Due to the long relaxation time, a temperature chamber was used to remove the heat generated by the test apparatus and provide insulation against external temperature variations, leading to a constant testing temperature of $23.00 \pm 0.01{ }^{\circ} \mathrm{C}$. The samples were initially subjected to nanoindentation at a peak load of $3 \mathrm{mN}$ and then the resultant relaxation of nanoindentation load was recorded for $1 \mathrm{~h}$.

The Wiechert model, a generalised Maxwell model, was used for describing the timedependant viscoelastic behavior. ${ }^{33,34}$ This model consists of an arbitrary number of springdashpot Maxwell elements connected in parallel and a constant factor, $E_{0}$, describing the contribution, which is independent of time. The relaxation modulus, $E_{r e l}$, at a time, $t$, is defined as:

$$
E_{r e l}(t)=\frac{F(t)}{A \varepsilon_{0}}=E_{0}+\sum_{i=1}^{n} E_{i} \exp \left(-\frac{t}{\tau_{i}}\right)
$$

where $F(t)$ is the force, $A$ is the contact area, $\varepsilon_{0}$ is the constant strain applied to the specimen during the stress relaxation test, $E_{i}$ are the relaxation coefficients for different relaxation times, $\tau_{i}$, and $E_{0}$ is the time-independent coefficient. The relaxation spectrum, which presents the coefficients, $E_{i}$, as a function of relaxation time was obtained by fitting the experimental data to the Wiechert model. At least nine measurements from each region (epoxy matrix with and without CNTs) were tested to obtain a statistical average value.

\section{Results and discussion}


The cross-section shown in this section is typical of the sample; one coherent example is presented with all the characterisation, including AFM, Raman and nanoindentation, performed on the same CNT-grafted fibre, to aid interpretation of the different methodologies.

\subsection{Growth of CNTs on fibres}

Earlier work ${ }^{11}$ has shown that the thickness of multi-walled CNTs grown on silica fibres can be controlled by varying the growth time. For this work, the length of the grafted CNT was chosen to be around $30 \mu \mathrm{m}$, both for convenience and to address the practically relevant lengthscales. This lengthscale is convenient as it allows a clear distinction of the CNTmodified region and provides a reasonable sample size for investigation by the various techniques. It is not yet clear what length of grafted nanotubes will prove to be most useful for hierarchical composites. However, in order to maximise interactions but not limit fibre packing excessively, it may be on the order of the primary fibre diameter, so at least 10 microns, but not the millimetre or even centimetre scale that can be achieved in some growth experiments. Shorter arrays tend to have poor alignment, whilst a grafted length around 30 microns provides a reasonable degree of radial alignment, and is likely to be relevant to hierarchical composite work in the field. The methodologies developed in this paper may be readily applied to longer or somewhat shorter grafted nanotubes. SEM images in Figure 1 illustrate that aligned, densely-packed CNTs were uniformly grown around the silica fibres, having a radial 'bottle brush' morphology, with a splitting pattern often observed during the growth of relatively long CNTs around cylindrical fibres. ${ }^{11,} 35$. The growth and characterisation of the specific CNT-grafted fibre batch used in this work was reported previously; ${ }^{11}$ the average CNT diameter was $27 \pm 7 \mathrm{~nm}$.

\subsection{AFM characterisation of cross-sections of model hierarchical composites containing}

\section{CNT-grafted silica fibres}

The polished cross-section of the model hierarchical epoxy composites containing CNTgrafted silica fibres was initially examined using AFM. Images shown in Figure 2 are 
representative of the specimen and can be reproduced at different magnifications. The topographic image (Figure 2a) shows the silica fibre slightly brighter, suggesting the fibre ends were exposed and approximately $50 \mathrm{~nm}$ above the polymer matrix region after the polishing process, as expected due to the greater hardness of the silica fibre; this phenomenon is a recognised effect of differential polishing between hard reinforcements and softer matrices. ${ }^{36}$ Different topography was observed in the polymer region around the fibre, presumably due to the presence of CNTs, which was confirmed by the Raman spectroscopy study as discussed in the next section. A statistical analysis of surface roughness of different regions was performed and the results are summarised in Table 1. The average and root mean square roughnesses of the CNT/epoxy region were 18 and $24 \mathrm{~nm}$, respectively, which is comparable to the intrinsic diameter of CNTs. These roughness values were about six times higher than that of the pure epoxy region, indicating that the presence of the CNTs had significantly increased the surface roughness, due to their intrinsically greater characteristic scale compared to the polymer network.

The phase-contrast image was generated simultaneously, based on the phase angle shifts during scanning, which often correspond in a complex way to changes in the local properties of the sample surface, such as variations in composition, topography, adhesion and viscoelasticity. ${ }^{37}$ Thus, the detection of phase angle shifts can provide enhanced contrast and better resolution, especially for heterogeneous and rough surfaces. ${ }^{38}$ Indeed, the boundaries of the primary fibre are particularly clear in the phase contrast image (Figure 2b). Similarly, apparent phase contrast was observed in the rough CNT-modified epoxy region.

\subsection{Distribution and orientation of CNTs in model hierarchical composites}

The distribution of the CNTs in the epoxy matrix was determined by performing Raman spectroscopy on the same embedded CNT-grafted fibre as presented in the previous section, although using a smaller scan area of $26 \times 26 \mu \mathrm{m}^{2}$. Typical spectra of the CNTs (Figure 3) were observed in the rough area surrounding the fibre, showing the characteristic, graphite 
lattice related $\mathrm{D}$ and $\mathrm{G}$ bands. ${ }^{39}$ The $\mathrm{D}: \mathrm{G}$ intensity ratio after embedding was the same as that of the as-produced CNTs, ${ }^{11}$ indicating the resin infusion and polishing processes had a negligible effect on crystallinity. The Raman spectra of the pure epoxy region agree well with literature results. ${ }^{40}$ Based on the intensity of the CNT D-band (around $1345 \mathrm{~cm}^{-1}$, refer to the dark grey range marked in Figure 3) and one of the epoxy signals (around $820 \mathrm{~cm}^{-1}$, the light grey range marked in Figure 3), Raman maps of CNTs (Figure 4a) and epoxy (Figure 4b) were generated, clearly presenting the distribution of CNTs in the matrix. The G band of CNTs, around $1580 \mathrm{~cm}^{-1}$, was not selected in this case since the matrix also showed Raman features in the range of $1550-1600 \mathrm{~cm}^{-1}$. The fibre appears dark in both cases, since no Raman signal for silica was detected in the selected ranges (refer to Figure 3). A couple of very bright spots in Figure $4 \mathrm{~b}$ are likely due to the strong fluorescence background from diamond particles which remained on the composite surface after polishing. Due to the strong, resonant enhancement of the CNT Raman signal, and their strong optical absorbance, the Raman signal for the epoxy is suppressed in the CNT-rich regions, leading to a relatively constant and dark region around the silica fibre in the epoxy map. Unfortunately, this effect precludes the quantitative calculation of local CNT concentration in the current case; the use of a more Raman-active matrix and calibrations for absorbance may allow quantitative distribution maps in the future. Here, the epoxy-rich regions are marked, qualitatively, by the dashed lines in Figure $4 \mathrm{~b}$, which correspond to the relatively dark regions in Figure 4a. The lack of complete radial symmetry can be attributed to splitting of the CNT array (indicated by the arrows in Figure 4a), which is a typical of CNTs grafted around cylindrical fibres, beyond a critical length, due to circumferential strains. ${ }^{11,35}$ The distribution of CNTs obtained using Raman spectroscopy is relatively consistent with the AFM images (Figure 2). Raman spectroscopy provides complementary data to the AFM study, confirming the interpretation of both imaging methods. AFM rapidly locates the CNT distribution based on topology and phase, but could potentially be affected by the grinding and polishing process. On the other 
hand, the Raman map is likely to be less susceptible to such effects, since the collection volume is deeper. The similarity of the imaging techniques provides confidence that that sample fabrication has not introduced significant artefacts.

It is widely accepted that the orientation and alignment of CNTs in a composite is important for improving the through-thickness performance of composites. ${ }^{41,}{ }^{42}$ Polarised Raman spectroscopy was performed to study the CNT orientation. A preliminary investigation was conducted on the as-produced CNT-grafted silica fibres using polarised incident laser irradiation. The laser power and integration times used to record the spectra in different regions were identical. As shown in Figure 5, higher signal intensity was always obtained when the laser polarisation direction was parallel to the nanotube axis. Moreover, no change of the D:G ratio was observed, suggesting this ratio is independent of the CNT orientation and alignment, in agreement with previously published data. ${ }^{43}$

Polarised Raman spectroscopy was applied to the same cross-sectional area of the composite specimen discussed above. Based on the characteristic D-band, Raman maps of CNTs were recorded at two different, orthogonal polarisation directions (Figure 6a-b). Both polarised Raman maps indicated the same distribution of CNTs in the polymer matrix, but with different contrast, indicating different orientations of CNTs in the specimen. A semiquantitative map of the local orientation was calculated by considering the maximum intensity ratio of the two Raman polarisations (Figure $6 a-b$ ); where $I_{\text {vertical }} / I_{\text {horizontal }}>1$, the map is plotted in shades of blue, where $I_{\text {horizontal }} / I_{\text {vertical }}>1$, it is plotted in shades of yellow. This approach to data presentation had been carefully selected to ensure that the contrast scales in a consistent manner in the two different orientations. Thus, at each point, the orientation of the CNTs can be deduced from the colour and contrast of the calculated Raman map (Figure 6c). CNTs with orientation between 0 and $45^{\circ}$ to the vertical polarisation direction are displayed blue (such as the region below the silica fibre); the contrast increases with increasing alignment to the vertical direction. Conversely, the CNTs oriented at between 45 and $90^{\circ}$ to the vertical 
polarisation direction are displayed yellow (such as the regions on both sides of the silica fibre); the contrast increases as the alignment approaches $90^{\circ}$. Both the $I_{\text {vertical }} / I_{\text {horizontal }}$ and $\mathrm{I}_{\text {horizontal }} / \mathrm{I}_{\text {vertical }}$ ratios for the silica fibre, epoxy and $45^{\circ}$-orientated CNTs were equal to 1 , resulting in the black regions in the map. It can be inferred qualitatively from this orientation study that the CNT alignment around the fibre was maintained after composite fabrication. However, the semi-quantitative map provides insight for further experiment optimisation, as well as theoretical studies. A fully quantitative approach would require rotation of either the sample or the polarisers, systematically, in small angular steps. Rotating the sample is difficult given the need to remain central on the primary fibre of interest, and the current equipment setup does not allow rotation of the polarisers. In any case, such an approach would create a very large volume of data and significant associated challenges, but could be used if specific orientation distributions were needed, perhaps for computational studies. The present approach of using two polarisation directions has been used for other nanocomposites, ${ }^{44}$ and offers a good compromise between the level of information obtained and the mapping efficiency. More generally, polarised Raman spectroscopy can provide information about the distribution and orientation of CNTs in hierarchical composites, and could be applied to a variety of hairy fibres and hierarchical composite systems consisting of components with different Raman characteristic signals. As an example, Raman maps showing the distribution of CNTs in a model hierarchical polyester matrix composite were obtained using the same method (Supporting information, Figure S1 and S2). It's worth noting that Raman spectroscopy does not distinguish local CNT orientation due to processing from intrinsic structural waviness; it's not even clear that there is a meaningful distinction at the resolution limit of the technique (around one micron), since shear/flow effects that control orientation are likely to bend the individual CNTs, and both effects will degrade mechanical reinforcement. ${ }^{45,46}$ Studies of the effect of pixel size on quantitative measures of alignment 
may shed light on the relative contributions of processing and intrinsic (growth) effects, in the future.

\subsection{Mechanical performance around carbon nanotube grafted silica fibres in model hierarchical composites}

Nanoindentation tests were conducted on the same embedded CNT-grafted fibre, in order to study the impact of grafted CNTs on the mechanical properties of the epoxy matrix surrounding the fibre. Figure $7 \mathrm{a}$ shows the topographic image of the specimen after nanoindentation; an array of $3 \times 20$ nanoindentation imprints can be clearly observed, covering regions within the silica fibre, the CNT-modified epoxy and the pure epoxy. Both the silica fibre and epoxy can be considered as isotropic materials. In the anisotropic CNT-modified region, the indentation direction is consistently perpendicular to the CNT orientation, i.e. in the "transverse direction" of the CNT-reinforced epoxy. The Oliver and Pharr method was developed for isotropic materials but has been widely applied to anisotropic materials, such as aligned CNT-reinforced nanocomposites ${ }^{47}$ and biomaterials ${ }^{48}$. Whilst anisotropic materials do demonstrate orientation-dependent indentation behaviour, ${ }^{49}$ the stress field is complex; the indentation modulus is likely to be an average value weighted in the direction of indentation ${ }^{48}$. The current work measures an effective 'indentation modulus' (as defined in the ISO 14577 standard) in the transverse direction of the aligned CNT-modified epoxy materials. Whilst different values would be expected if the indentation was parallel to the CNT direction, the approach nevertheless consistently maps the reinforcing efficiency around the fibre. Representative load-displacement curves in different regions are shown in Figure $7 \mathrm{~b}$. Theoretically, for a constant force, a smaller displacement would imply a harder specimen. The lowest nanoindentation depth was observed as expected for the silica fibre, which is the hardest material in this system. Introduction of CNTs into epoxy reduced the nanoindentation displacement, suggesting the anticipated reinforcing effect of the matrix. The typical indentation depth was around $300 \mathrm{~nm}$ (corresponding to contact area around $6 \mu \mathrm{m}^{2}$ ) for the 
CNT/epoxy region and much larger than the scale of the surface roughness (refer to Table 1, around $20 \mathrm{~nm}$, about $6.7 \%$ of the indentation depth), confirming that the sample polishing is sufficient. A further quantitative study of the hardness and indentation modulus in the transverse direction was performed as a function of the distance from the fibre (Figure 7c-d). Note that distances were measured to the centre of each set of three indentations, introducing a small additional uncertainty for the points very close to the primary fibre. Both hardness and indentation modulus were fairly constant in the range of 35 to $90 \mu \mathrm{m}$ from the fibre, i.e., within the pure epoxy region, given that the length of the grafted CNTs was around $30 \mu \mathrm{m}$ as determined by SEM, AFM, and the Raman maps. Apparent increases in hardness and indentation modulus were observed when approaching the silica fibre, which can be attributed to the increasing local CNT content in the matrix. Ideally, a 1/radius dependency might be expected for a pure radial distribution of CNTs, assuming a linear dependence of hardness on CNT content; in reality, variations in orientation or local CNT content (due to the splitting discussed above) will occur. The surface roughness of the CNT-modified epoxy region (refer to Table 1) and the variation in local CNT content is the likely source of the larger standard deviations nearer the primary fibre. The average hardness and elastic modulus of the epoxy were $0.13 \pm 0.01$ and $2.23 \pm 0.03 \mathrm{GPa}$, respectively, in good agreement with literature data on same type of bisphenol-based epoxy resin. ${ }^{19,20}$ The hardness and modulus of the epoxy matrix surrounding the CNT grafted silica fibres, i.e. the CNT-modified epoxy, increased up to 0.17 \pm 0.01 and $2.77 \pm 0.15 \mathrm{GPa}$, demonstrating the benefits of CNT-reinforcement. The improvement was about $28 \%$ for the hardness and $24 \%$ for the modulus, which was again comparable to literature nanoindentation data on CNT-reinforced epoxy nanocomposites. ${ }^{18,19}$, 21, 22 Improvements of hardness and modulus in the range $5-75 \%$ have been reported on mixing 0.5-5 wt $\%$ single-walled, ${ }^{19,21}$ coiled $^{22}$ and aligned ${ }^{18}$ CNTs in epoxy. The extent of the reinforcing effect of CNTs on the composite mechanical properties may be affected by various factors, such as intrinsic surface and mechanical properties, ${ }^{2}$ alignment ${ }^{47,} 50$ and 
waviness $^{46,51}$ of the CNTs. It is worth pointing out that compared to the in-plane modulus at the same CNT loading, the current geometry is expected to measure a lower indentation modulus (in the transverse direction) based on an inverse rule of mixtures. ${ }^{52}$ Nanoindentation tests parallel to the CNTs are more difficult in the current sample geometry, although work on related, flat, aligned arrays is well-known. ${ }^{47}$

The effect of CNTs on the viscoelastic properties of the epoxy matrix were studied by relaxation tests. The typical normalised force as a function of time in different regions is presented in Figure 8a. It is clear that the CNT-modified epoxy region exhibited a shallower slope than the pure epoxy region, suggesting slower relaxation in the presence of CNTs. By fitting the experimental data to the Wiechert model, ${ }^{33,34}$ relaxation time spectra were obtained and are compared in Figure 8b. For the pure epoxy region, the peak value appeared around $1 \mathrm{~s}$ and the coefficient values in the time frame of $10^{-1}$ to $10^{3} \mathrm{~s}$ were higher than those obtained for the epoxy region containing CNTs, indicating a faster relaxation. The CNT-modified epoxy region possessed the same peak position around $1 \mathrm{~s}$, which suggested that the primary relaxation mechanism was unchanged. However, a shoulder at about $10^{2} \mathrm{~s}$ could be seen in the spectra; the broadening effect or shift to longer relaxation times may be attributed to the restricted molecular mobility of polymer caused by interaction with the CNTs. The immobilisation of polymer surrounding the CNTs could be considered as an additional reinforcing component, which may cause mechanical stiffening in the system. ${ }^{53}$ Similar broadening effects have been observed in dynamic mechanical thermal analysis (DMTA) measurements of $\tan \delta$ for macroscopic nanocomposite samples. ${ }^{54}$ Furthermore, the timeindependent coefficient, $E_{0}$, for the CNT-rich regions was higher than that for the pure epoxy, suggesting a higher modulus, in agreement with the nanoindentation tests.

\section{Conclusions}

The combined AFM/Raman technique is a useful tool for the characterisation of hierarchical composites, providing information regarding CNT distribution and orientation around the 
primary reinforcing fibres. CNT maps can be successfully created based on characteristic Raman signals and their intensity ratios obtained using different laser polarisation directions. Nanoindentation tests revealed the reinforcing effect of CNTs on the epoxy matrix in the vicinity of the fibres, showing $28 \%$ and $24 \%$ improvements of hardness and indentation modulus in the transverse direction. The local stiffening of the matrix surrounding the fibres may reduce the tendency for fibre microbuckling under longitudinal compression, which is critical to overall composite performance. This study demonstrates the efficiency of the CNTs as local nanoreinforcement and provides critical information that should be useful for future modelling of CNT-based hierarchical composites. In addition, a slower stress relaxation was observed in the epoxy region containing CNTs, which may be due to restricted molecular mobility of the matrix. The immobilisation of polymer surrounding the CNTs may provide additional mechanical stiffening for the composites, which could be strongly dependent on the CNT/polymer interactions. Further relaxation studies of CNTs with different surface chemistries might help to improve the understanding of these interactions at a local level. The toolkit that has been developed and demonstrated, here, should find further application in the study of both CNT-reinforced nanocomposites and CNT-based hierarchical composite systems. In the case of hierarchical systems in which the CNTs are directly mixed into matrix before infusion or prepregging, the orientation maps will help to clarify the effects of local matrix flow around and within fibre tows.

Whilst the current work has demonstrated useful qualitative mapping of CNT distribution and semi-quantitative characterisation of CNT orientation, further quantification would be useful, particularly for advancing the modelling and theory of hierarchical systems. The long term ambition is to develop the techniques presented, and others, to allow full mapping of local concentration, orientation, waviness, and a wider range of resulting (nano)composite properties. As discussed above, the matrix system could be redesigned to aid the determination of CNT concentration, possibly in combination with other mapped optical 
spectroscopies. More detailed polarisation measurements would clarify local orientation; images/maps collected at different magnifications would shed light on CNT waviness. Sections polished parallel to the primary fibres would provide further information about CNT uniformity and orientation, as well as performance anisotropy. The use of a variety of AFM derivatives could allow mapping of thermal and electrical conductivities, as well as other mechanical characteristics. A wide range of techniques are either already available or currently emerging that have the potential to shed light on the complex multiscale phenomena at the heart of hierarchical composites.

\section{Acknowledgements}

The authors gratefully acknowledge the funding provided by dstl (Defence Science and Technology Laboratory, Porton Down, UK) and the EU FP 7 programme StorAGE. SGK acknowledges the research funding from the European Research Council under the European Community's Seventh Framework Programme (FP7/2007-2013)/ERC advanced grant agreement no. [227950]. 


\section{References}

1. J. N. Coleman, U. Khan, W. J. Blau and Y. K. Gun'ko, Carbon, 2006, 44, 1624-1652.

2. M. Shaffer and J. Sandler, in Processing and Properties of Nanocomposites, ed. S. G. Advani, World Scientific, 2006, pp. 1-59.

3. M. Moniruzzaman and K. I. Winey, Macromolecules, 2006, 39, 5194-5205.

4. M. T. Byrne and Y. K. Gun'ko, Adv. Mater., 2010, 22, 1672-1688.

5. $\quad$ X.-L. Xie, Y.-W. Mai and X.-P. Zhou, Mat. Sci. Eng. R 2005, 49, 89-112.

6. A. R. Bunsell and J. Renard, in Fundamentals of fibre reinforced composite materials, Taylor \& Francis, 2005, pp. 1-17.

7. H. Qian, E. S. Greenhalgh, M. S. P. Shaffer and A. Bismarck, J. Mater. Chem., 2010, 20, 4751-4762.

8. K. T. Hsiao, in Processing and Properties of Nanocomposites, ed. S. G. Advani, New Jersey, NJ [etc.] : World Scientific, 2007, pp. 141-189.

9. L. T. Qu, Y. Zhao and L. M. Dai, Small, 2006, 2, 1052-1059.

10. H. Qian, A. Bismarck, E. S. Greenhalgh, G. Kalinka and M. S. P. Shaffer, Chem. Mater., 2008, 20, 1862-1869.

11. H. Qian, A. Bismarck, E. S. Greenhalgh and M. S. P. Shaffer, Carbon, 2010, 48, $277-$ 286.

12. H. Qian, A. Bismarck, E. S. Greenhalgh and M. S. P. Shaffer, Composites Part A, 2010, 41, 1107-1114.

13. R. J. Sager, P. J. Klein, D. C. Lagoudas, Q. Zhang, J. Liu, L. Dai and J. W. Baur, Compos. Sci. Technol., 2009, 69, 898-904.

14. F. H. Zhang, R. G. Wang, X. D. He, C. Wang and L. N. Ren, J. Mater. Sci., 2009, 44, 3574-3577.

15. H. Qian, A. Bismarck, E. S. Greenhalgh and M. S. P. Shaffer, Compos. Sci. Technol., 2010, 70, 393-399.

16. Q. Zhao and H. D. Wagner, Phil. Trans. R. Soc. A, 2004, 362, 2407-2424.

17. R. Haggenmueller, H. H. Gommans, A. G. Rinzler, J. E. Fischer and K. I. Winey, Chem. Phys. Lett., 2000, 330, 219-225.

18. H. Lee, S. Mall, P. He, D. L. Shi, S. Narasimhadevara, Y. Yeo-Heung, V. Shanov and M. J. Schulz, Composites Part B, 2007, 38, 58-65.

19. A. K. Dutta and D. Penumadu, J. Mater. Res., 2004, 19, 158-164.

20. D. C. Lagoudas, P. R. Thakre and A. A. Benzerga, Nanoindentation of CNT reinforced epoxy nanocomposites Alexandroupolis, Greece, 2006.

21. X. D. Li, H. S. Gao, W. A. Scrivens, D. L. Fei, X. Y. Xu, M. A. Sutton, A. P. Reynolds and M. L. Myrick, Nanotechnology, 2004, 15, 1416-1423.

22. X.-F. Li, K.-T. Lau and Y.-S. Yin, Compos. Sci. Technol., 2008, 68, 2876-2881.

23. P. M. Nagy, D. Aranyi, P. Horváth, P. Pötschke, S. Pegel and E. Kálmán, Internet Electron. J. Mol. Des., 2006, 5, 135-143.

24. K. Koziol, M. Shaffer and A. Windle, Adv. Mater., 2005, 17, 760-763.

25. K. L. A. Chan and S. G. Kazarian, Nanotechnology, 2010, 21, 445704.

26. A. Ureña, J. Rams, M. D. Escalera and M. Sánchez, Compos. Sci. Technol., 2005, 65, 2025-2038.

27. W. C. Oliver and G. M. Pharr, J. Mater. Res., 1992, 7, 1564-1583.

28. G. M. Pharr, W. C. Oliver and F. R. Brotzen, J. Mater. Res., 1992, 7, 613-617.

29. B. J. Briscoe, L. Fiori and E. Pelillo, J. Phys. D-Appl. Phys., 1998, 31, 2395-2405.

30. Y. F. Zhang, S. L. Bai, X. K. Li and Z. Zhang, J. Polym. Sci. Part B: Polym. Phys., 2009, 47, 1030-1038.

31. B. Ashrafi and P. Hubert, Compos. Sci. Technol., 2006, 66, 387-396.

32. S. Chung, S. Makhar, H. Ackler and S. Park, Electron. Mater. Lett., 2006, 2, 175-181. 
33. C. Machiraju, A.-V. Phan, A. W. Pearsall and S. Madanagopal, Comput. Meth. Prog. Bio., 2006, 83, 29-33.

34. S. Ronan, T. Alshuth and S. Jerrams, Kgk-Kautschuk Gummi Kunststoffe, 2007, 60, 559-563.

35. A. Y. Cao, V. P. Veedu, X. S. Li, Z. L. Yao, M. N. Ghasemi-Nejhad and P. M. Ajayan, Nat. Mater., 2005, 4, 540-545.

36. E. Greenhalgh, in Failure analysis and fractography of polymer composites, Woodhead Publishing Limited, 2009, pp. 23-106.

37. F. Z. Fang, Z. W. Xu and S. Dong, Meas. Sci. Technol., 2008, 19, 055501.

38. L. C. Sawyer, D. T. Grubb and G. F. Meyers, Polymer microscopy, 3rd ed., Springer., 2007.

39. A. Jorio, M. A. Pimenta, A. G. Souza, R. Saito, G. Dresselhaus and M. S. Dresselhaus, New Journal of Physics, 2003, 5, 17.

40. M. Asquier, P. Colomban and V. Milande, J. Raman Spectrosc., 2009, 40, 1641-1644.

41. V. P. Veedu, A. Y. Cao, X. S. Li, K. G. Ma, C. Soldano, S. Kar, P. M. Ajayan and M. N. Ghasemi-Nejhad, Nat. Mater., 2006, 5, 457-462.

42. E. J. Garcia, B. L. Wardle, A. J. Hart and N. Yamamoto, Compos. Sci. Technol., 2008, 68, 2034-2041.

43. D. Fischer, P. Pötschke, H. Brünig and A. Janke, Macromol. Symp., 2005, 230, $167-$ 172.

44. P. Pötschke, H. Brünig, A. Janke, D. Fischer and D. Jehnichen, Polymer, 2005, 46, 10355-10363.

45. H. Cebeci, R. G. de Villoria, A. J. Hart and B. L. Wardle, Compos. Sci. Technol., 2009, 69, 2649-2656.

46. F. T. Fisher, R. D. Bradshaw and L. C. Brinson, Appl. Phys. Lett., 2002, 80, $4647-$ 4649.

47. E. J. Garcia, A. J. Hart, B. L. Wardle and A. H. Slocum, Adv. Mater., 2007, 19, 21512156.

48. J. G. Swadener, J.-Y. Rho and G. M. Pharr, J. Biomed. Mater. Res., 2001, 57, 108-112.

49. A. B. Mann, in Nanotribology and nanomechanics: an introduction 2nd Edition, ed. B. Bhushan, Springer-Verlag Berlin Heidelberg, 2008, p. 635.

50. $\quad$ L. Ci, J. Suhr, V. Pushparaj, X. Zhang and P. M. Ajayan, Nano Lett., 2008, 8, 27622766.

51. V. Anumandla and R. F. Gibson, Composites Part A, 2006, 37, 2178-2185.

52. M. R. Piggott, Load-Bearing Fiber Composites, 2nd Edition, Kluwer Academic Publisher, Norwell, USA, 2002.

53. A. Eitan, F. T. Fisher, R. Andrews, L. C. Brinson and L. S. Schadler, Compos. Sci. Technol., 2006, 66, 1162-1173.

54. M. Q. Tran, J. T. Cabral, M. S. P. Shaffer and A. Bismarck, Nano Lett., 2008, 8, 27442750 . 

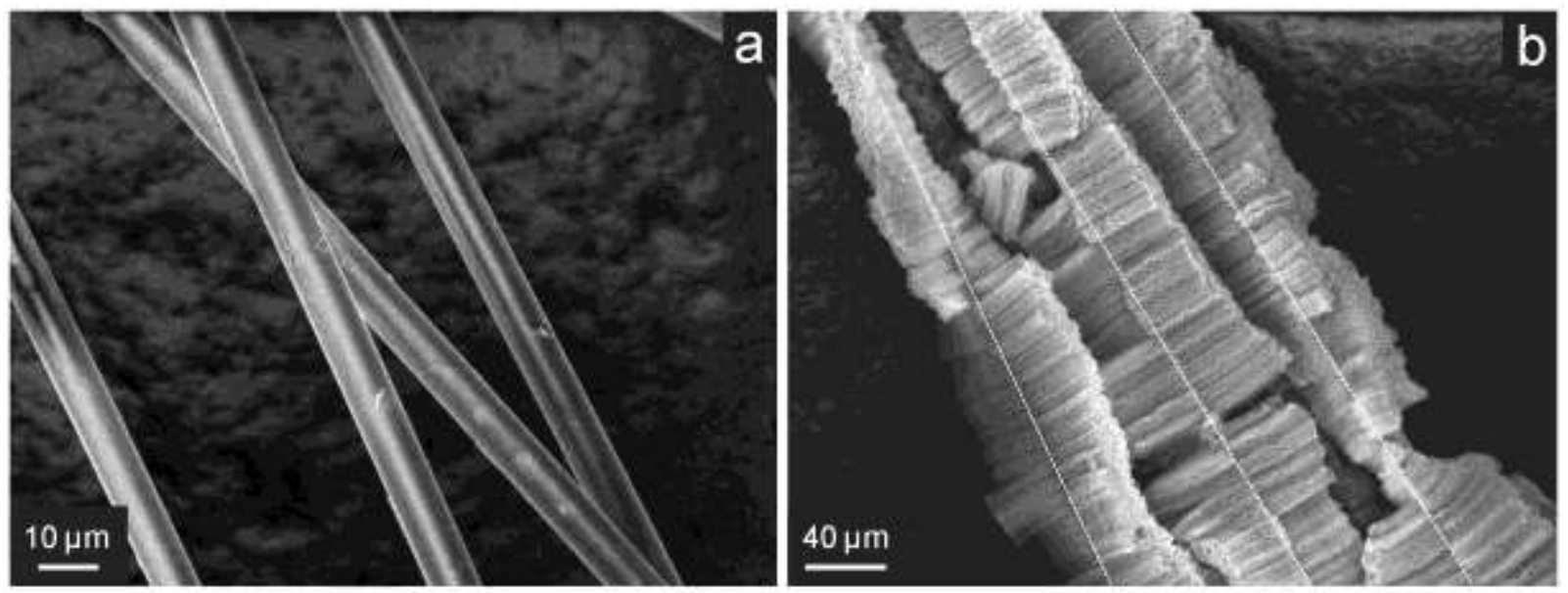

Figure 1. SEM images of silica fibres (a) before and (b) after the CNT growth reaction via injection chemical vapour deposition. The positions of the silica fibres are indicated by dashed lines in (b).
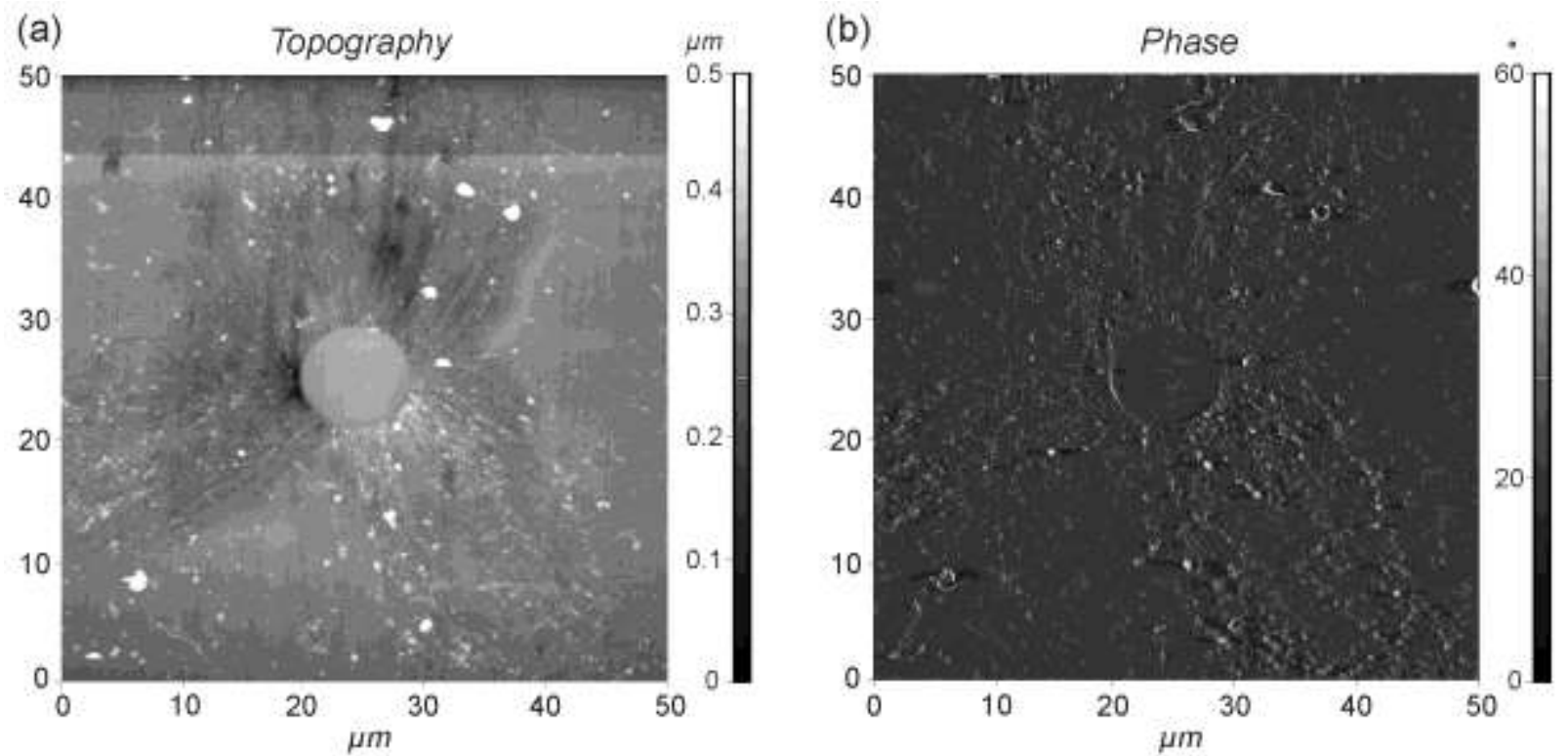

Figure 2. (a) Topographic and (b) phase-contrast AFM images recorded in the $50 \times 50 \mu \mathrm{m}^{2}$ area of the polished cross-section of a model hierarchical epoxy composite containing CNTgrafted silica fibres. 


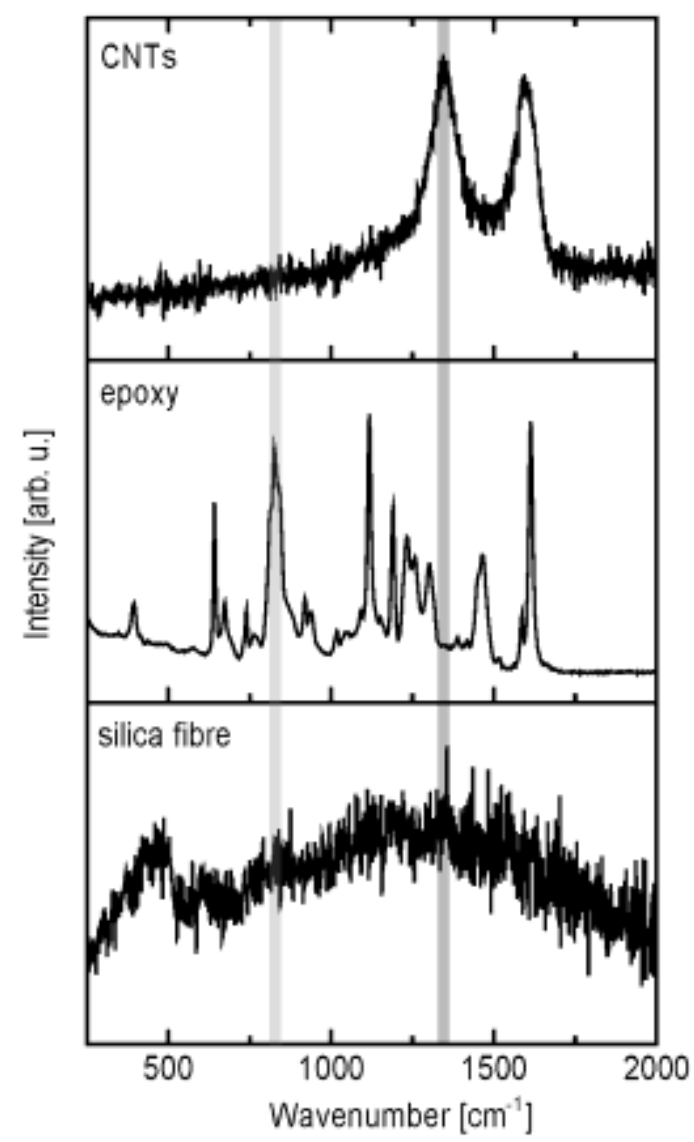

Figure 3. Raman spectra recorded in different regions of the polished cross-section of a model hierarchical epoxy composite containing CNT-grafted silica fibres. The characteristic signals of CNTs and epoxy used to produce Raman mappings are highlighted in dark grey and light grey, respectively.

(a)

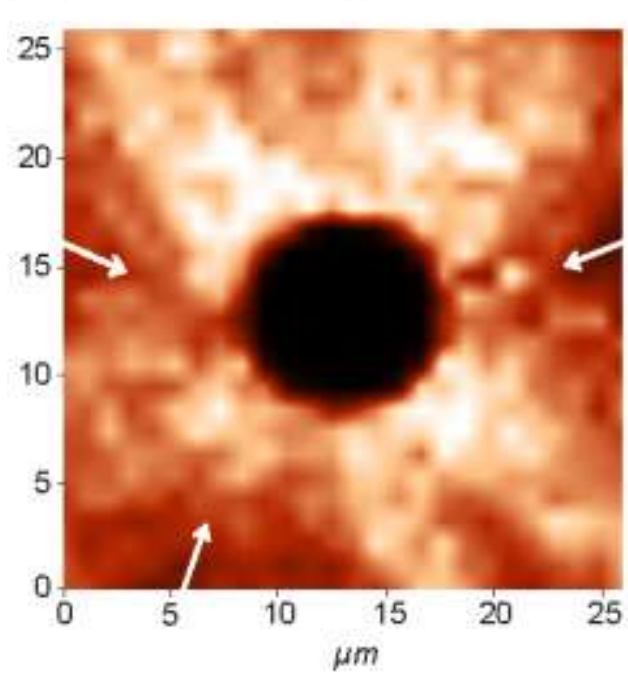

(b)

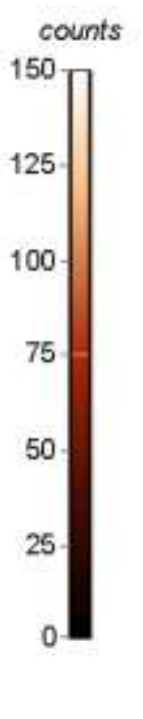

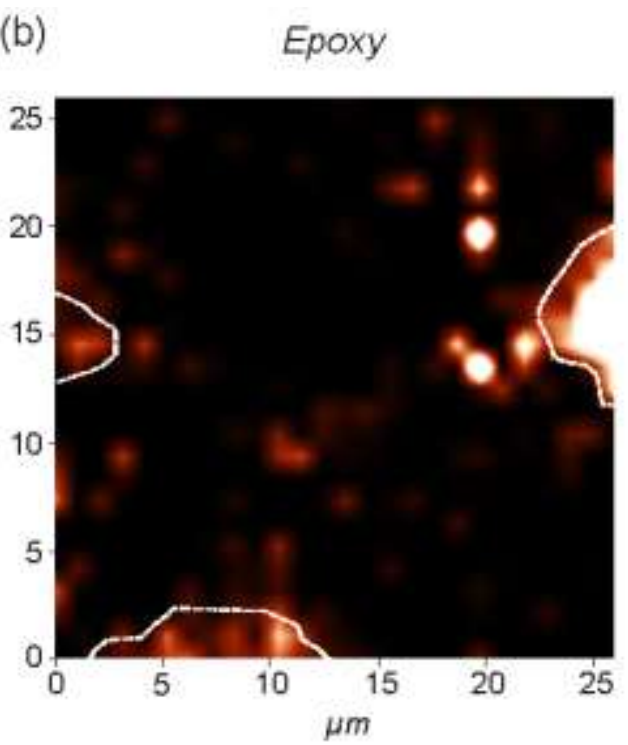

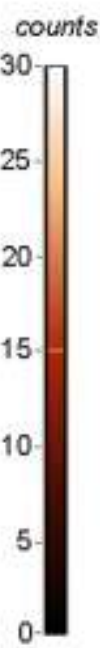

Figure 4. Raman mappings recorded in the $26 \times 26 \mu \mathrm{m}^{2}$ area of the polished cross-section of a model hierarchical epoxy composite containing CNT-grafted silica fibres based on the characteristic signals of (a) CNTs and (b) epoxy. The splitting positions of CNT arrays are indicated by the arrows in (a). The epoxy-rich regions are represented by the dashed lines in (b). 

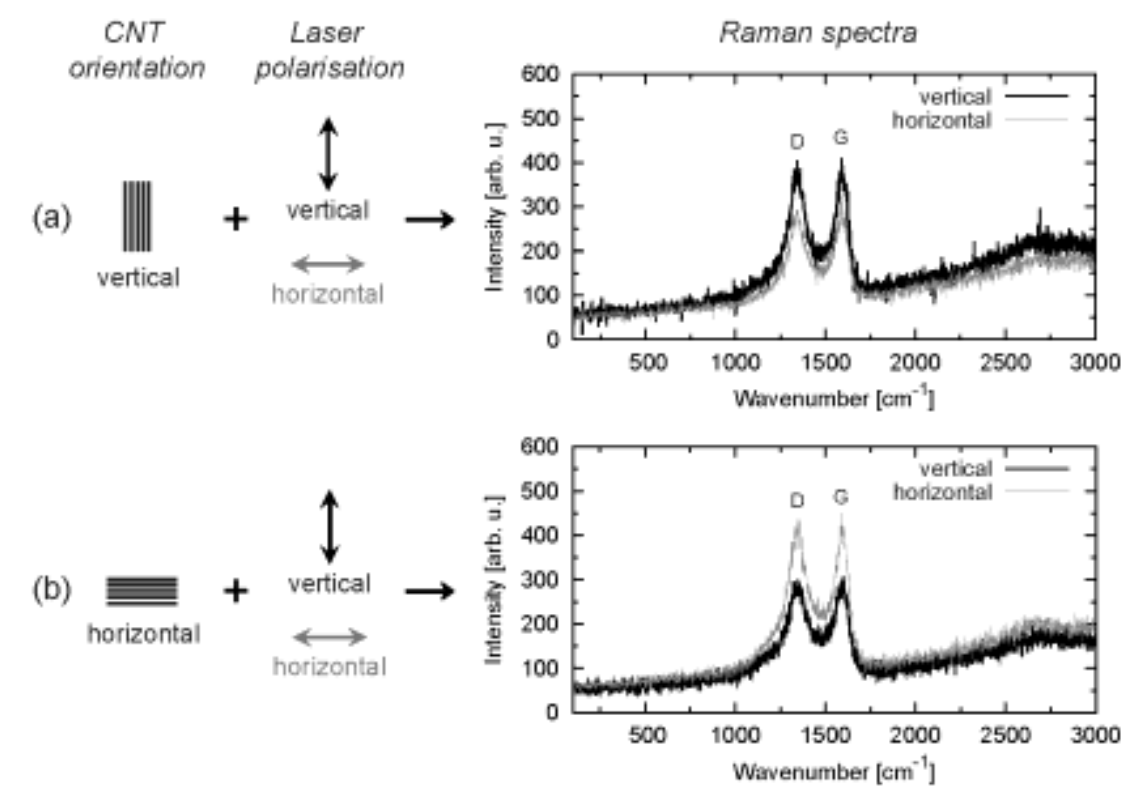

Figure 5. Raman spectra of (a) vertically and (b) horizontally aligned CNTs using polarised incident laser irradiation.
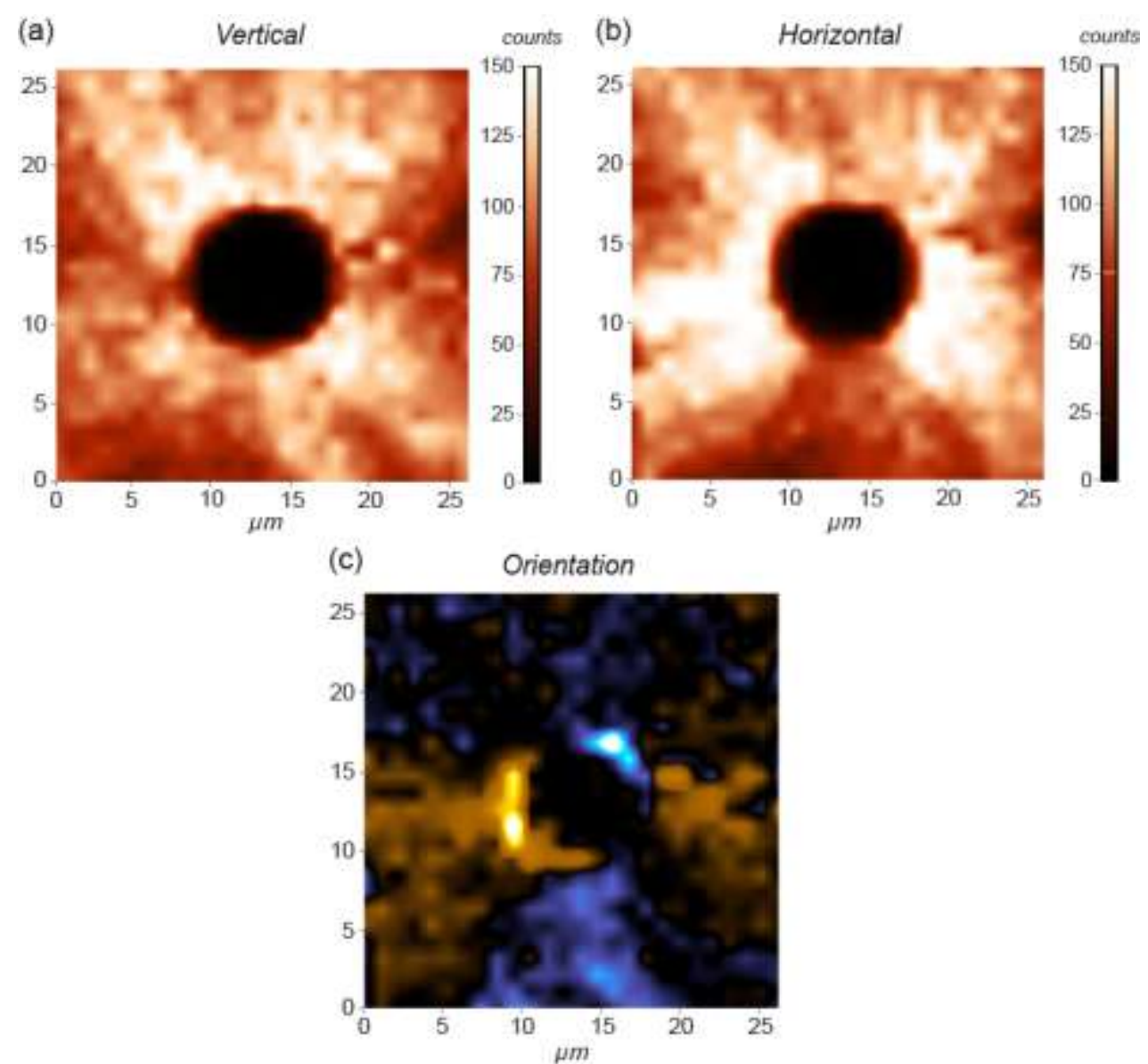

Figure 6. Polarised Raman maps recorded in a $26 \times 26 \mu \mathrm{m}^{2}$ area of the polished cross-section of a model hierarchical epoxy composite containing CNT-grafted silica fibres using (a) vertically- and (b) horizontally-polarised incident laser. (c) Calculated Raman map of CNT orientation. Vertically- and horizontally-orientated CNTs were represented in (c) by blue and yellow, respectively. 

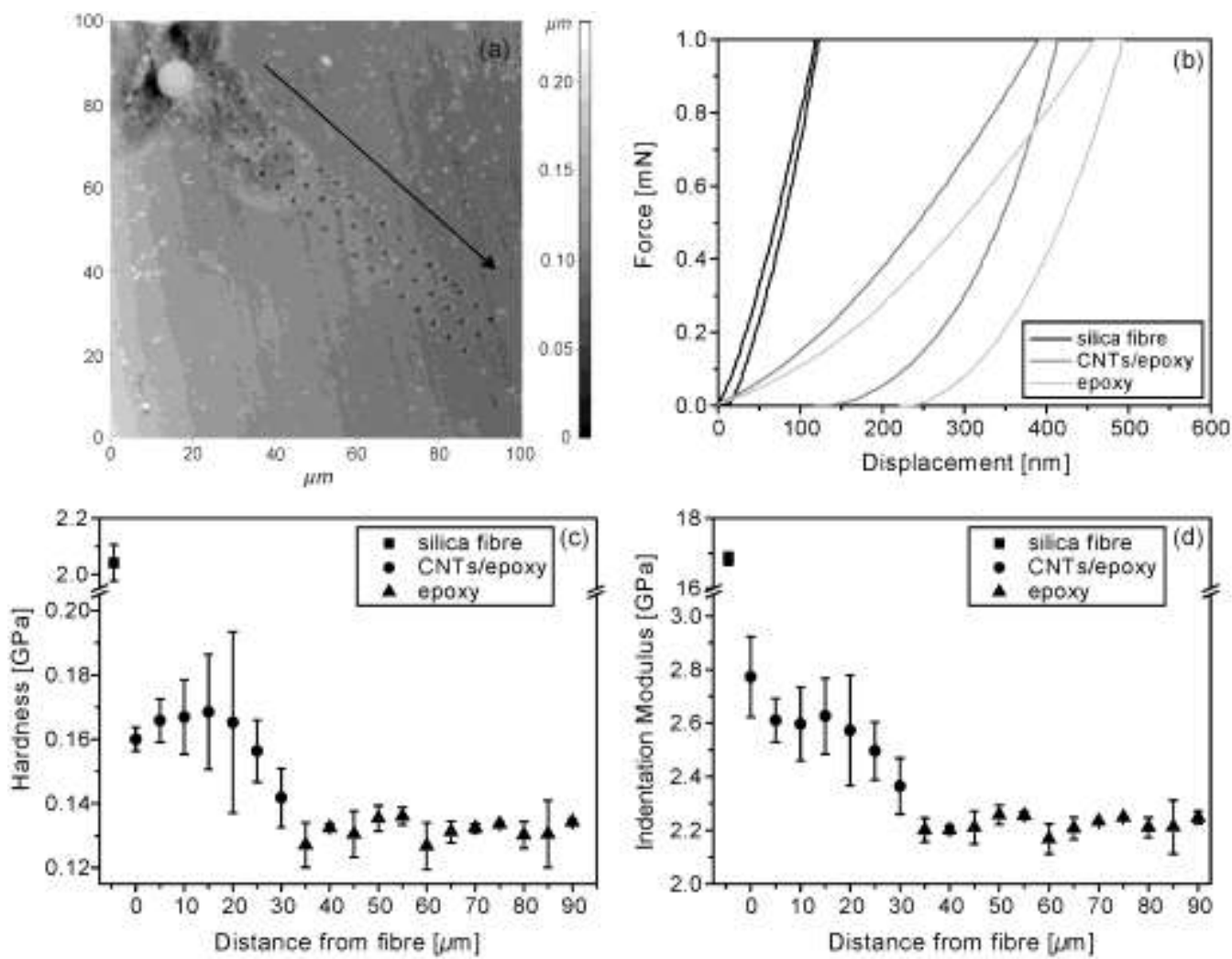

Figure 7. (a) Topographic image of an array of $3 \times 20$ nanoindentation imprints on the polished cross-section of a model hierarchical epoxy composite containing CNT-grafted silica fibres. (b) Typical force-displacement curves corresponding to nanoindentations performed in different regions. (c) Hardness and (d) indentation modulus as a function of the distance from fibre. The average and standard deviation values in (c) and (d) were based on three indentations. 

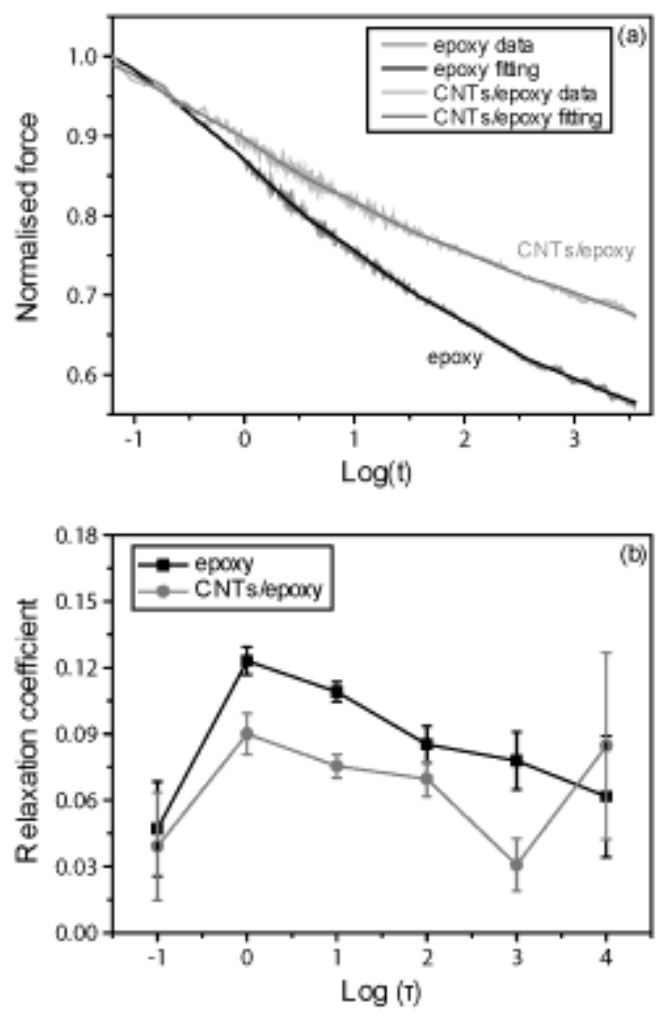

Figure 8. (a) Relaxation test results of different regions: experimental data and Wiechert model fit. (b) Relaxation time spectra, i.e. the coefficients of the fitted Wiechert model. 
Table 1. Surface roughness of different regions of the polished cross-section of a model hierarchical epoxy composite containing CNT-grafted silica fibres. The CNT/epoxy values were obtained based on the measurements conducted in the CNT-modified region next to the silica fibre. The standard deviations are shown in parentheses.

\begin{tabular}{ccc}
\hline Region & Average roughness $S_{a}[\mathrm{~nm}]$ & Root mean square roughness $S_{q}[\mathrm{~nm}]$ \\
\hline Silica fibre & $8.6(0.2)$ & $9.7(0.2)$ \\
CNT/epoxy & $18.1(4.8)$ & $24.4(7.3)$ \\
Epoxy & $2.8(0.1)$ & $4.0(0.1)$ \\
\hline
\end{tabular}

